\title{
Multiphase Design Exploration Method for Lightweight Structural Design: Example of Vehicle Mounted Antenna-Supporting Structure
}

\author{
Sungwoo Jang', Chung Hyun Goh ${ }^{23}$, and Hae-Jin Choi ${ }^{1, *}$ \\ 1 School of Mechanical Engineering, Chung-Ang University, 84, Heukseok-ro, Dongjak-gu, Seoul, 156-756, South Korea \\ 2 Next Generation Energy Safety Research Institute, Chung-Ang University, 84, Heukseok-ro, Dongiak-gu, Seoul, 156-756, South Korea \\ 3 Systems Realization Laboratory, University of Oklahoma, Norman, OK 73019, USA \\ \# Corresponding Author / E-mail: hjchoi@cau.ac.kr, TEL: +82-2-820-5787, FAX: +82-2-814-9476
}

KEYWORDS: Inductive design exploration method, Lightweight design, Materials and product design, Robust design, Topology optimization

\begin{abstract}
In this study, we design a lightweight antenna-supporting structure for a vehicle-mounted radar system using a multiphase design exploration method. In the first phase of the approach, structural topology within a given design domain is optimized in order to reduce the weight and increase the structural integrity applying various design scenarios, which provides several preliminary structural layouts. Inspecting the commonalities and differences of the preliminary layouts, key shape (i.e., geometry) parameters in an initial layout to be further explored are chosen. In the second phase, design variables, the chosen shape parameters and properties of its material are concurrently explored to satisfy the given system requirements. For the concurrent design exploration of the materials and structures, the inductive design exploration method is employed for obtaining feasible ranged sets of design variables, instead of a single optimum solution. In this way, a designer may simply make a robust choice among the feasible sets under various sources of uncertainties and multiple performance requirements.
\end{abstract}

Manuscript received: March 28, 2015 / Revised: May 7, 2015 / Accepted: May 31, 2015

\section{NOMENCLATURE}

VMRS: Vehicle-mounted radar system

ATC: Air traffic control

IDEM: Inductive design exploration method

IMPD: Integrated materials and product design

SIMP: Solid isotropic microstructure with penalty

HD-EMI: Hyper-Dimensional error margin index

$r$ : Radius [mm]

$t$ : Thickness [mm]

$\rho$ : Density $\left[\mathrm{kg} / \mathrm{m}^{3}\right]$

$\sigma_{y}$ : Yield strength [MPa]

$M$ : Total mass of antenna-supporting structure [kg]

S.F.: Safety factor of antenna-supporting structure

$\mathbf{B}_{\mathrm{j}}$ : Discrete point vector on a constraint boundary

$\mathbf{B}_{\mathbf{j}}^{\mathbf{i}}$ : Projected vector of $\mathbf{B}_{\mathbf{j}}$ along the $\mathbf{i}$ direction on the nearest

boundary of an output range $\mathbf{u}_{\mathrm{i}}$ : Unit vector along the $\mathbf{i}$ direction

mean: Vector of the mean of an output range

\section{Introduction}

The vehicle-mounted radar system (VMRS) is currently one of the most popular systems employed on the battlefield because it improves the survivability and mobility of the troops. A VRMS typically operates on the roof or shelter of vehicles. Thus, the important parameters determining its performance, such as the antenna beam-width, operational frequency, and transmitted wavelets, depend on the payload limitations of a launch vehicle. An antenna-supporting structure in the VRMS is an essential component, of which primary function is to support the antenna and direct the radiation in space. Thus, the structure, in addition to its main function of supporting the antenna, should rotate along the azimuth axis and permit angular displacement of the antenna in 

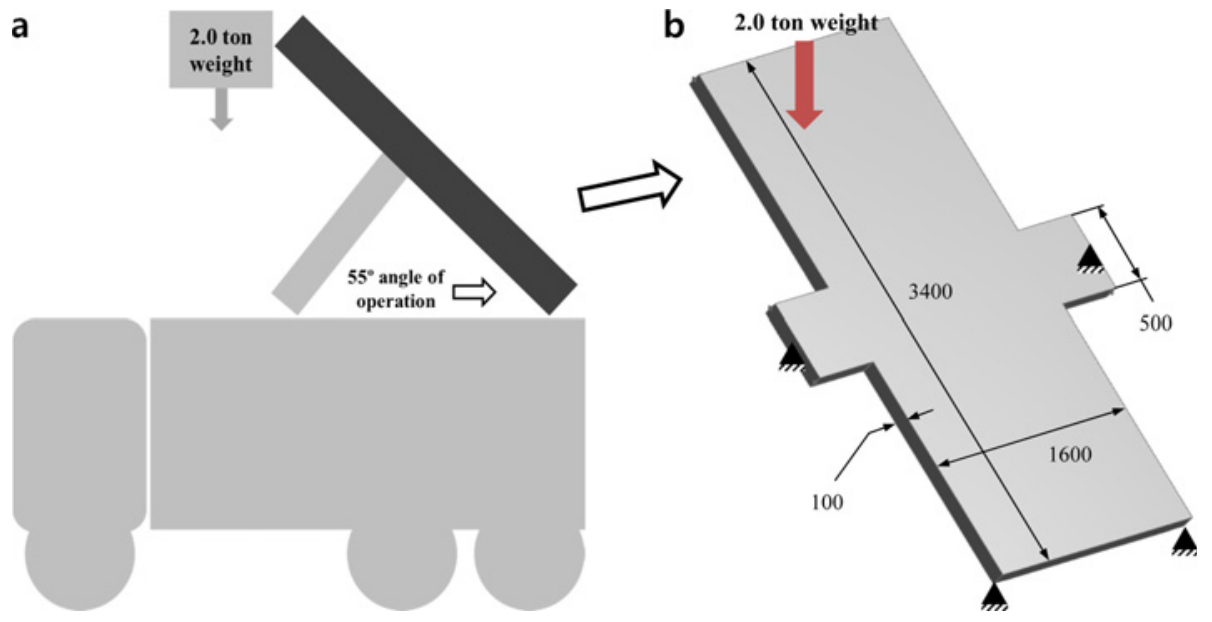

Fig. 1 (a) Design domain of antenna-supporting structure (b) Given design domain

elevation. ${ }^{1}$ For the antenna-supporting structures, high-strength, lightweight materials such as radar-absorbing materials are typically used in order to satisfy multiple system requirements, e.g., the system must resist high strength and achieve rapid maneuverability.

A number of optimization techniques are applied to solve these multi-objective design problems. Especially, methods for new topologies with lightweight materials can be found in the literatures, such as, 3D printing technology with various topology set-ups, ${ }^{2}$ polymer materials for use in lightweight vehicles, ${ }^{3}$ shape optimization of large-scale wind turbine, ${ }^{4}$ and so forth. Nevertheless, many literatures consider only topology optimization or shape (size) optimization itself.

In addition, material selection or single optimum solution is not always possible for achieving multiple system-level performances. For example, a complex design problem may be decomposed into a number of sub-problems, each of which may have independent/dependent design variables with different goals, disciplines, scales, etc. Also, the presence of uncertainty via design hierarchy makes more difficult to identify system-wide design solution.

In this study, we employ a multiphase design exploration method for designing topology and geometric parameters of the antenna-supporting structures, addressing aforementioned issues. Furthermore, our method focuses not only integrating optimizations of structural topology and parameters, but exploring feasible material candidates with the consideration of uncertainty. Therefore, a designer can quickly identify satisfactory regions of robust solutions within which the final structural shape and material can be chosen to meet multiple system requirements. Application of our design approach to a given design problem is explained in detail in Section 2.

\section{Design Approach for Lightweight Structures}

The design domain of an antenna-supporting structure is shown in Fig. 1. The structure is clamped at four positions to support a 2-ton radar at a $55^{\circ}$ angle of operation. Fig. 1(b) shows the given initial domain to be designed, where the design goals are to make the structure lightweight as well as to ensure its high safety performance. In this problem, we assume that only the weight of radar is considered as a given loading condition, while thermal stress or dynamic

\section{Topology optimization}

- Non-parametric optimization

- Initial structural layout

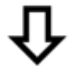

Parameterization

- Key shape parameters

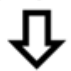

Integrated Materials and Product Design

- Concurrent design of material and layout

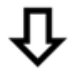

Inductive Design Exploration Method

- Feasible material and geometry

- Robust solution region

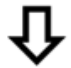

\section{Selection of best design}

Fig. 2 Design flow in multiphase design exploration method

deflection are not considered. In order to attain the goals, we employ a new design method, termed the multiphase design exploration method. Its procedure is shown in brief in Fig. 2.

First, we perform topology optimization on the predefined design domain with given structural boundary conditions and load definition. The main objective of this topology optimization is to determine the optimum material distribution within the design space that will achieve mass reduction while satisfying constraints. ${ }^{5}$ As an outcome of topology optimization, an initial structural layout can be drawn, to which more detailed design exploration is applied.

Whereas the topology optimization is a non-parametric optimization technique, it is necessary to parameterize appropriate design variables in the next phase. Here, we parameterize the geometry parameters based on the initial layout obtained by topology optimization, as well as the material properties of the structure. This approach of selecting geometry parameters and material properties as design variables has its roots in the integrated materials and product design (IMPD) approach. ${ }^{6-8}$ IMPD is an innovative design approach that features in concurrently designing the structure as well as its materials to enhance the system performance. Unlike conventional material-selection-based design, in which constitutive materials of a product are preselected from a 


\begin{tabular}{cccccc}
\hline Objective & Constraint & Objective & Constraint & Objective & Constraint \\
\hline $\begin{array}{c}\text { Minimize } \\
\text { Volume }\end{array}$ & $\begin{array}{c}\text { Strain energy } \\
\leq 50000 \mathrm{~mJ}\end{array}$ & $\begin{array}{c}\text { Minimize } \\
\text { strain energy }\end{array}$ & $\begin{array}{c}\text { Volume } \\
\leq 30 \%\end{array}$ & $\begin{array}{c}\text { Minimize } \\
\text { Volume }\end{array}$ & $\begin{array}{c}\text { Equivalent stress } \\
\leq 10 \mathrm{MPa}\end{array}$ \\
\hline a & b & & C & \\
\end{tabular}

Fig. 3 Topology optimization under various combinations of objectives and constraints; (a) Minimize volume with strain energy constraint of $\leq 50000 \mathrm{~mJ}$ (b) Minimize strain energy with permissible amount of volume of $\leq 30 \%$ (c) Minimize volume with stress constraint of $\leq 10 \mathrm{MPa}$

materials database, IMPD allows designers to customize the materials to meet design requirements.

Once the design variables have been defined, the inductive design exploration method (IDEM) is employed to obtain the final design specification. IDEM is an efficient multilevel robust design strategy for exploring the feasible range of design specifications in a step-by-step, top-down (inductive) manner. ${ }^{6,713}$ In IDEM, feasible regions of predefined design variables (not an optimum point) are identified so that design selection can be performed within these feasible regions. IDEM also takes account of the effect of uncertainty in design variables that leads to performance variability, therefore enabling exploration of robust solution regions.

The remainder of this paper is organized as follows. Section 3 describes the procedures of the multiphase design exploration method for designing the lightweight antenna-supporting structure. Section 4 discusses the design results followed by the conclusion in Section 5.

\section{Multiphase Design Exploration Method}

\subsection{Phase 1: Topology Optimization}

Topology optimization has been widely applied in the area of structural optimization problems because the performance of structures is greatly influenced by their topology. ${ }^{9-12}$ In topology optimization, the material layout is optimized within the specified design area such that the resulting layout meets the performance targets, e.g., maximizing structural stiffness while using the permitted amount of material.

In order to perform topology optimization in a finite element analysis (FEA) environment, the given design space (Fig. 1(b)) is modeled with mesh elements $50 \mathrm{~mm}$ in size. The 2-ton antenna weight as located in Fig. 1 and boundary conditions of fixed supports are also applied. The self-weight of the antenna support structure is not considered in the analysis since the weight of the support structure is much less than antenna itself and the 2-ton equivalent point load is very conservative value comparing with various real loading conditions. The given loading and boundary conditions resemble a 2-D plane problem; therefore, 2-D topology optimization is suitable in this problem. Aluminum alloy is given as the constitutive material for performing

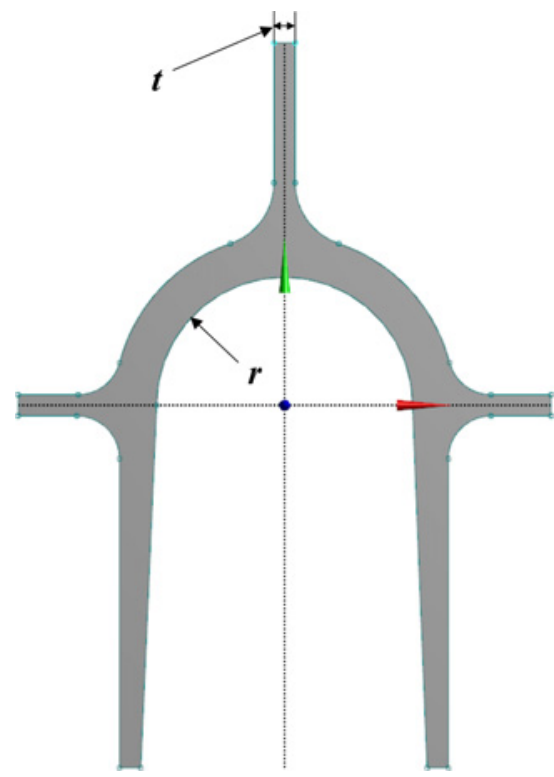

Fig. 4 Initial layout of the antenna-supporting structure

static analysis. A number of topology optimizations are performed based on various combinations of objective functions and constraints since our purpose of topology optimization is to find initial structural layout to be further fine-tuned. The values for constraints of strain energy, volume, and equivalent stress are not given as fixed values but roughly determined after performing a number of optimizations. This is a reasonable approach since we need to find the commonality of shapes determined by various topology optimization scenarios at this time. Design optimization under specified requirements and goals will be performed in Phase 2 discussed in Section 3.2.

Fig. 3 shows the derived optimum topologies based on various combinations of objective functions and constraints. It is observed that the topology results appear to have a slingshot shape in common, which leads to an initial layout of the antenna supporting structure. Based on the shapes shown in Fig. 3, an initial layout is designed to be manufactured in conventional production processes. In the remodeling process, key shape parameters to be found at the next phase of the design exploration method must be determined.

As shown in Fig. 4, two geometry parameters, $r$ and $t$, are selected 


\section{Property level}

\section{Performance level}

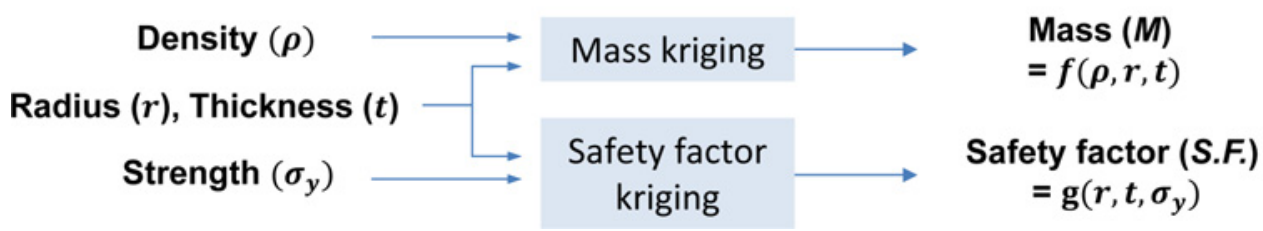

Fig. 5 Analysis models mapping between the property (geometry) level and the system performance level
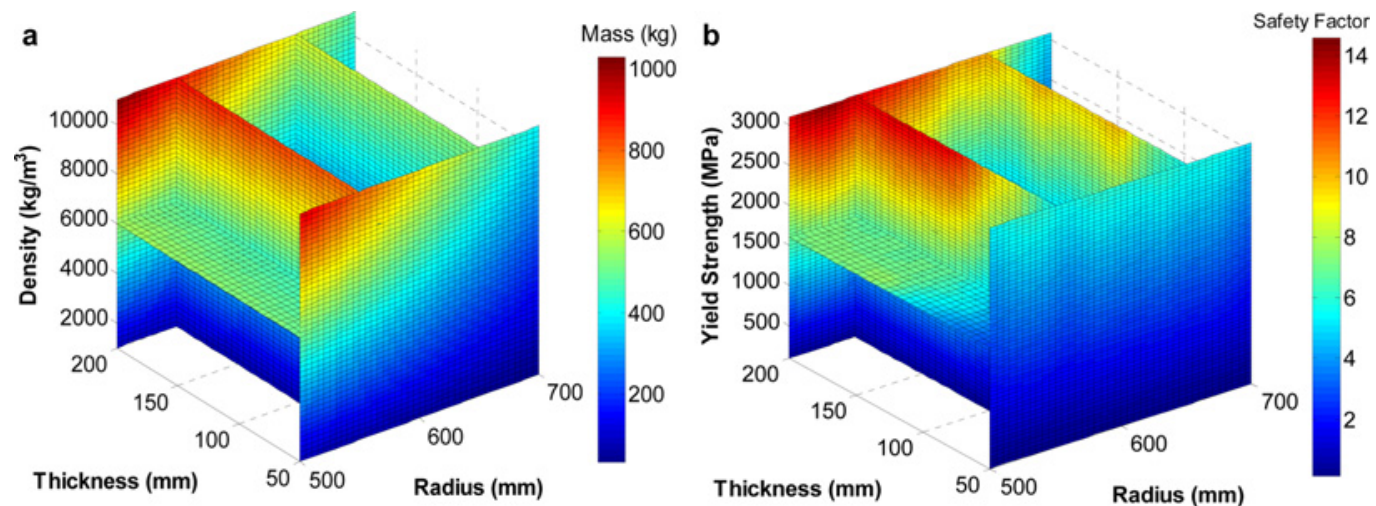

Fig. 6 Volumetric slices of the kriging models for (a) mass and (b) safety factor

as key shape parameters that represent the commonality of the shapes shown in Fig. 3. The radius, $r$, determines the thickness of the body frame and $t$ does the thickness of the neck where being loaded. They vary depending on problem formulations in the topology optimization.

Other parameters selected as the design variables in this study are the material properties of density $(\rho)$ and strength $\left(\sigma_{y}\right)$. This approach of choosing geometry parameters and material properties as design variables is based on the aforementioned IMPD which aims to concurrently design materials and product layout.

\subsection{Phase 2: Inductive Design Exploration Method (IDEM) \\ 3.2.1 Employed Mapping Models}

The analysis models need to be organized in order to apply IDEM to the given design problem. Fig. 5 shows the analysis models mapping between the property (geometry) level and the system performance level. As mentioned earlier, two geometry parameters ( $r$ and $t$ ), density $(\rho)$, and strength $\left(\sigma_{y}\right)$ are chosen as design variables at the property level. As the performance parameters, the total mass $(M)$ of the antenna-supporting structure and the safety factor (S.F.), which is defined as the strength $\left(\sigma_{y}\right)$ divided by 3 times of the equivalent (von Mises) stress derived from the FEA model of the frame structure, are selected. The mass performance is determined on the basis of the density and the geometry parameters of the structure. Furthermore, the safety factor performance is determined on the basis of the geometry parameters and material strength.

A rough design space must be adequately defined to explore the feasible regions in IDEM. The ranges of the two considered geometry parameters are set to $500 \mathrm{~mm} \leq r \leq 700 \mathrm{~mm}$ and $50 \mathrm{~mm} \leq t \leq 200 \mathrm{~mm}$, based on the initial layout shown in Fig. 4. The design range of material properties is specified based on "Ashby plot for materials selection" 14 in which feasible materials can be found. The ranges of strength and density are set to $100 \mathrm{MPa} \leq \sigma_{y} \leq 3000 \mathrm{MPa}$ and $1000 \mathrm{~kg} / \mathrm{m}^{3}$ $\leq \rho \leq 10000 \mathrm{~kg} / \mathrm{m}^{3}$, respectively, in order to cover a wide range of material categories, such as metals and composites.

The two mapping models are the mass kriging model and the safety factor kriging model. The adoption of a kriging model is necessary in this study since it can surrogate a large number of simulation iterations. ${ }^{15}$ A general kriging model is expressed as,

$$
y(x)=f(x)^{T} \beta+z(x)
$$

where $x$ denotes input design parameters, $f(x)^{T} \beta$ is a regression term to globally approximate the design space, and $Z(x)$ is the localized deviation from sample data.

The geometry parameters and density are used as input variables for the mass kriging model in order to predict the mass within the defined design space. Similarly, the safety factor kriging model can estimate the safety factor based on the geometry of the structure and the strength of its material. These kriging models are constructed based on sample simulation results (i.e., sample points) randomly generated by the Latin hypercube sampling method. ${ }^{16}$ In consideration of the wide range of design variables at the property level, 50 sample sets of input variables are generated for each kriging model and are evaluated by the simulation models. Based on the evaluated samples, the kriging models interpolate all the sample points, thereby predicting the overall performance responses. Secondorder polynomial regression models were used to globally fit the performance behaviors with Gaussian correlation function, the mostly preferred correlation function. Fig. 6 shows the two developed kriging models for the mass and safety factor. As mentioned, the mass and safety factor have three input variables for each, therefore we express the magnitudes of the cube responses (mass and safety factor) in RGB color space in the form of volumetric slices. From Fig. 6(a), it can be seen that the mass response is more significantly 


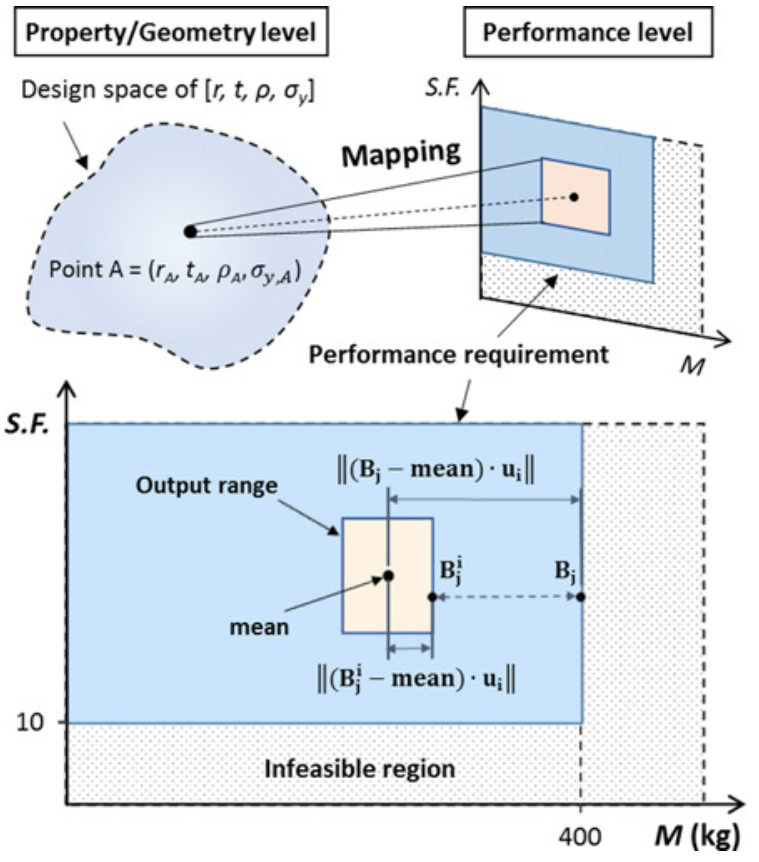

Fig. 7 Calculation of HD-EMI at point A

affected by the radius ( $r)$ than by thickness $(t)$ in the region where the density is approximately greater than $6000 \mathrm{~kg} / \mathrm{m}^{3}$. This may indicate that if one selects a material whose density is greater than $6000 \mathrm{~kg} / \mathrm{m}^{3}$, the radius is the only parameter that would reduce the structural weight. Similarly, the yield strength mainly affects the safety factor as well as the thickness of the neck where loading is applied, which is more sensitive to the safety factor than to the radius.

\subsubsection{Design Exploration Steps}

In this section, we explain three steps for designing the antennasupporting structure using the IDEM.

Step 1: Define a rough design space at the property level and generate discrete points within the space. The intervals between these discrete points are set to $10 \mathrm{~mm}, 10 \mathrm{~mm}, 500 \mathrm{~kg} / \mathrm{m}^{3}$, and $300 \mathrm{MPa}$ for $\mathrm{r}, \mathrm{t}, \rho$, and $\sigma_{y}$ respectively. A suitable interval size can be determined to tradeoff between the computation time and the solution accuracy.

Step 2: Evaluate the generated discrete points using the employed mapping models. As mentioned earlier, the mass and safety factor kriging models are considered as the mapping models between the property and the performance level. In order to consider the effect of uncertainty in simulation results due to idealization, $\pm 10 \%$ of outputs (performance variability) of each kriging model are assumed. Thus, the evaluation of a discrete point produces a range of outputs. These evaluated discrete points with the corresponding output ranges are stored for identifying the feasible regions in the next step.

Step 3: Sequentially identify the feasible regions at the property level to satisfy the given performance requirements. The feasible regions at property level consist of feasible points whose evaluated output ranges are located within a region (or regions) at performance level that satisfies the system requirements. In IDEM, these feasible points are determined based on the hyper dimensional error margin indices (HD-EMIs). ${ }^{6,7}$ The calculation of HD-EMIs is expressed in Eq. (2) and illustrated in Fig. 7.
Table 1 Design problem formulation

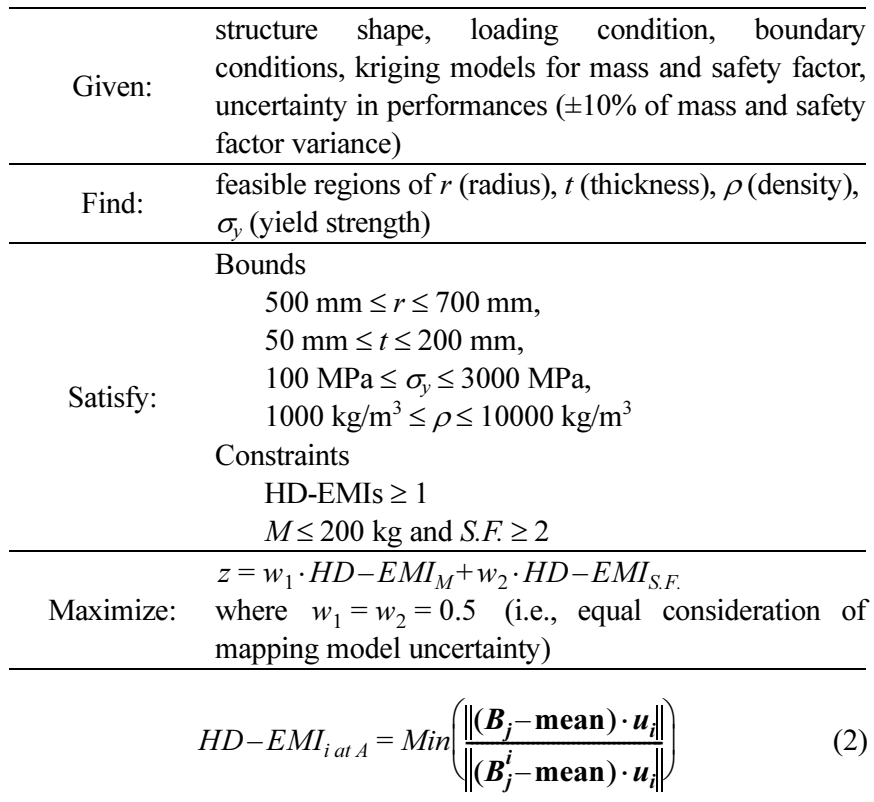

where $\boldsymbol{B}_{j}$ is a discrete point vector on a constraint boundary, mean is a vector of the mean of an output range, $\boldsymbol{u}_{i}$ is an unit vector along the $\boldsymbol{i}$ direction, and $\boldsymbol{B}_{\boldsymbol{j}}^{\boldsymbol{i}}$ is a projected vector of the $\boldsymbol{B}_{\boldsymbol{j}}$ along the $\boldsymbol{i}$ direction on the nearest boundary of an output range. An HD-EMI of a discrete point indicates the quantitative closeness of its output range to the nearest feasible region boundary, i.e., the robustness of a design point to not violate the performance constraints under its variability. An HDEMI greater than unity for a discrete point implies that its output range lies within the constraint boundary of the performance requirements, therefore making it a feasible point. With increasing HD-EMIs, the output range moves further away from the constraint boundary which will be more robust. However, if the mean of the output range lies outside the constraint boundary, the HD-EMI will take the value of -1 .

A design problem to select the most robust design solution can be formulated as shown in Table 1. In order to select a feasible point of $\left[r, t, \rho, \sigma_{y}\right]$ that will satisfy goals with the highest robustness, the objective function to be maximized is formulated as the equally weighted sum of HD-EMIs for mass and safety factor.

\section{Results and Discussion}

The described steps of the IDEM are coded and executed in MATLAB $^{\circledR}$. The feasible regions in property space that meet the two performance requirements (i.e., $M \leq 200 \mathrm{~kg}$ and $S . F . \geq 2$ ) are identified and stored in a database. These feasible regions consist of sets of fourdimensional feasible points expressed as $\left[r, t, \rho, \sigma_{y}\right]$, and the evaluated HD-EMI at each point is also stored. The points drawn in Figs. 8(a) and 8(b) indicate the feasible ranges in the $r, t$, and $\rho$ space when $\sigma_{y}$ is set as $3000 \mathrm{MPa}$ and $400 \mathrm{MPa}$, respectively. The magnitude of HD-EMI at each feasible point is expressed with the RGB color in the figures. Higher HD-EMI points (points in red) within the feasible region will result in output ranges located further away from the two required performance bounds, namely, higher robustness. Similarly, the points drawn in Figs. 8(c) and 8(d) indicate the feasible regions in the $r, t$, and $\sigma_{y}$ space with the fixed $\rho$ of $2500 \mathrm{~kg} / \mathrm{m}^{3}$ and $3000 \mathrm{~kg} / \mathrm{m}^{3}$, respectively. 

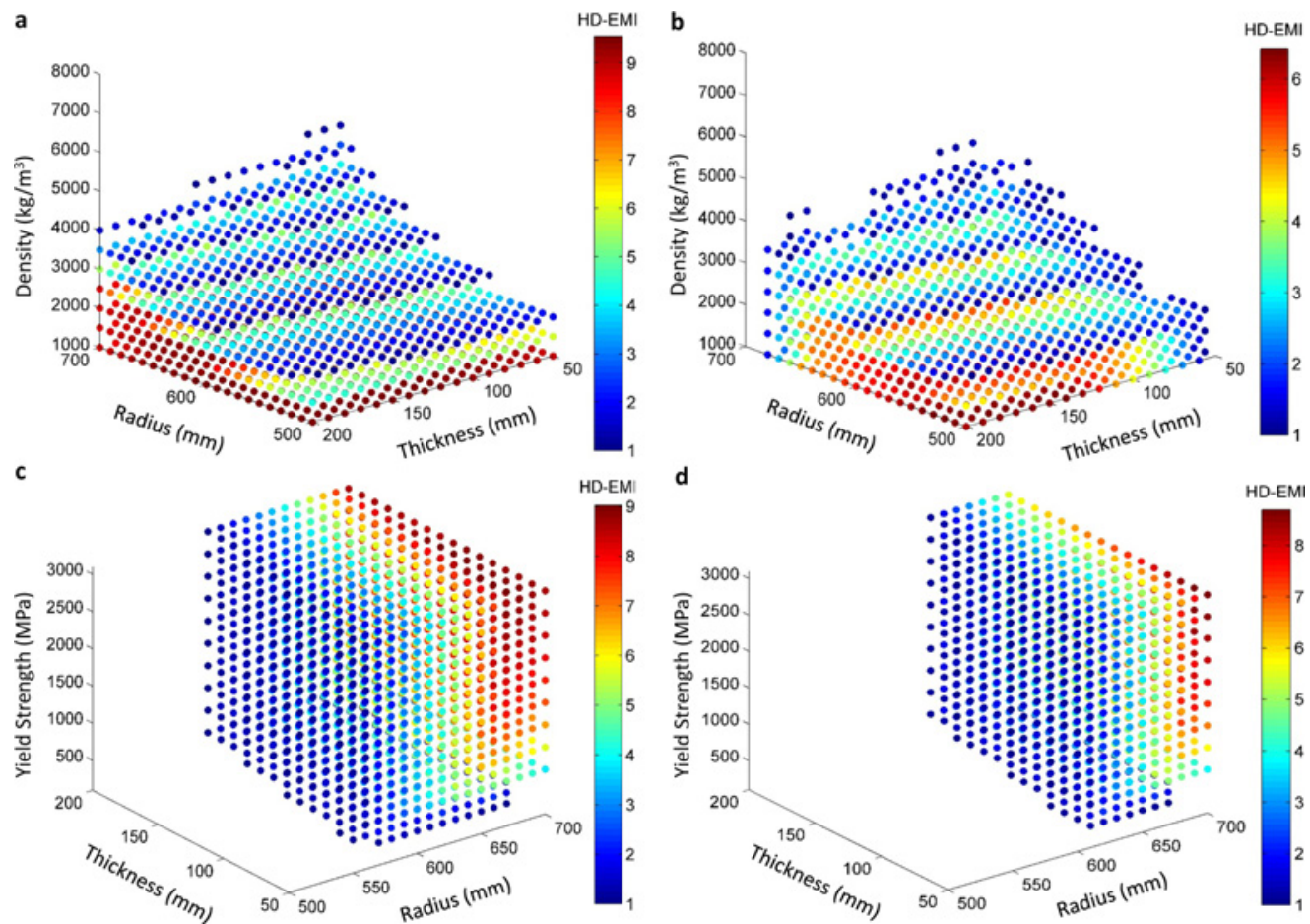

Fig. 8 Feasible region of geometry and density at (a) $\sigma_{y}=3000 \mathrm{MPa}$, (b) $\sigma_{y}=400 \mathrm{MPa}$; feasible region of geometry and strength at (c) $\rho=2500 \mathrm{~kg} / \mathrm{m}^{3}$, and (d) $\rho=3000 \mathrm{~kg} / \mathrm{m}^{3}$

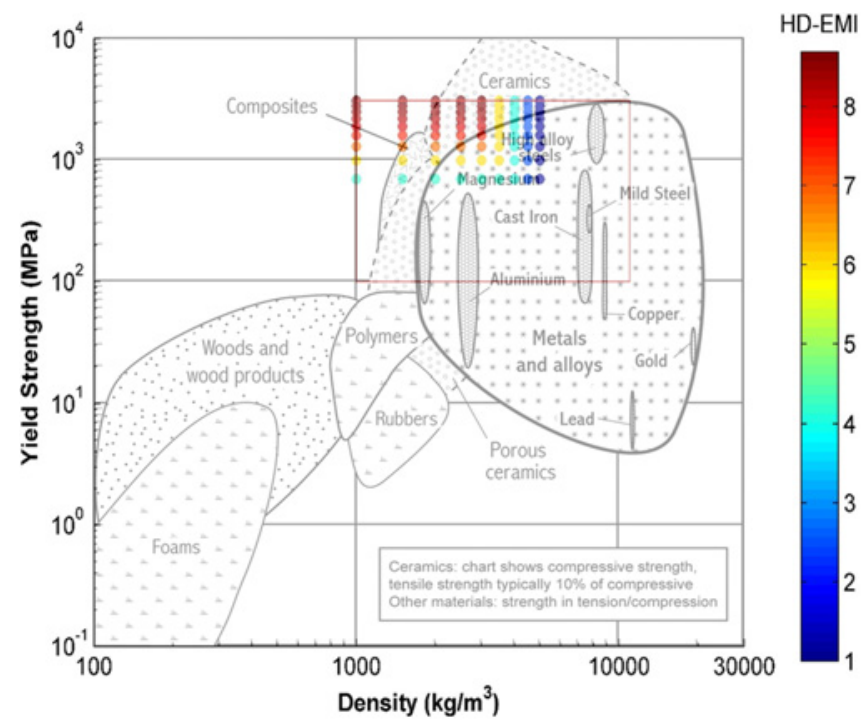

Fig. 9 Feasible region of material properties with a fixed geometry $(r=700 \mathrm{~mm}, t=60 \mathrm{~mm})$ in a density-strength Ashby map ${ }^{14}$

Based on the feasible regions in the property space as shown in Fig. 8 , one can strategically select a design specification. Therefore, the IDEM is beneficial for decision making in that it identifies the ranged sets of feasible points with indices of robustness, but not a single optimum solution. Feasible regions in material property $\left(\rho\right.$ and $\sigma_{y}$ ) space may be obtained by fixing the geometry design variables, since the feasible ranges of density and strength vary with changing geometry design variables. Fig. 9 shows the feasible region of the material properties with fixed geometry $(r=700 \mathrm{~mm}, t=60 \mathrm{~mm})$. We select the geometry design specifications that yield the largest feasible region in the space of $\rho$ and $\sigma_{y}$ with the given performance requirements to have largest number of available material candidates to select.
Table 2 Results of a selected set of desing specifications

\begin{tabular}{|c|c|c|c|c|}
\hline \multirow{2}{*}{ Geometry } & Radius [mm] & \multicolumn{3}{|c|}{700} \\
\hline & Thickness [mm] & \multicolumn{3}{|c|}{60} \\
\hline \multirow{2}{*}{ Material property } & Density $\left[\mathrm{kg} / \mathrm{m}^{3}\right]$ & \multicolumn{3}{|c|}{1600} \\
\hline & Yield strength [MPa] & \multicolumn{3}{|c|}{1400} \\
\hline \multirow{2}{*}{ HD-EMIs } & HD-EMImass & \multicolumn{3}{|c|}{10} \\
\hline & HD-EMIsafety factor & \multicolumn{3}{|c|}{6.8} \\
\hline \multirow{4}{*}{ Performances } & Mass $[\mathrm{kg}]$ & Min & Mean & Max \\
\hline & & 51.01 & 56.68 & 62.35 \\
\hline & Safety factor & Min & Mean & Max \\
\hline & & 6.22 & 6.91 & 7.60 \\
\hline
\end{tabular}

As shown in Fig. 9, the feasible points projected on the Ashby plot identify the candidate material choices, which indicates that a designer can select appropriate materials to be used, such as ceramics and composites, for satisfying the performance requirements. As aforementioned, this way brings a designer more design freedom and delays commitment to the predetermined materials, thereby increasing possibilities of decision making from the early stages of design process. Fig. 9 shows only the compressive strength of ceramics and it cannot be considered for a load-bearing structural material. Then, a designer can select a composite among the feasible materials, a design specification may be one of the feasible points on the range for composites, e.g., $\rho=1600 \mathrm{~kg} / \mathrm{m}^{3}$ and $\sigma_{y}=1400 \mathrm{MPa}$. The performance upon selection of this design specification can be computed using the kriging models, as presented in Table 2. The design results show that each of the performance requirements is sufficiently satisfied under the effect of uncertainty (i.e., 10\% assumed error in the simulation models in this study). HD-EMIs for mass and safety factor are also calculated. Both HD-EMIs are greater than unity that indicates the achievement of robustness under uncertainty. By choosing the design solutions within the identified feasible regions as performed in this study, we can 
manipulate the selection of design variables to negotiate between improving system performances and achieving the robustness of the solution in this multi-objective design problem, before a final design selection is validated in later design process.

\section{Conclusions}

This paper presents a hierarchical, multiphase design exploration method for designing an antenna-supporting structure of a VMRS, as well as the material of this structure. First, topology optimization is performed to determine optimal structures in the FEA environment. The resulting layout becomes the design concept before its shape and material are further tuned. Once an initial layout of the antenna frame structure is defined in the first phase, the geometry parameters (i.e., shape parameters) and properties of the constitutive material of the structure are parameterized as design variables to be explored in the next phase. The IDEM is employed for identifying the feasible regions of these design variables in the second phase. The IDEM yields not a single design result but rather ranged robust solution sets, which gives designers freedom in decision-making. By selecting a design specification of high HD-EMI, we can find a robust solution in presence of uncertainty. The feasible regions of the two considered geometry parameters, density, and strength are identified in order to satisfy the performance requirements of mass and safety factor as an outcome of the structure design. Finally, an effective, efficient method for selecting materials is established under given conditions of geometry and required properties. Combination of the Ashby plot for materials selection with exploration using the IDEM lends designers flexibility in selecting materials that would satisfy multiple systemlevel performance requirements.

\section{ACKNOWLEDGEMENT}

We thank support from LIG Nex1. This research was supported by Basic Science Research Program through the National Research Foundation of Korea (NRF) funded by the Ministry of Education (NRF-2014R1A1A2059731). This work was also supported by the Human Resources Program in Energy Technology of the Korea Institute of Energy Technology Evaluation and Planning (KETEP) granted financial resource from the Ministry of Trade, Industry \& Energy, and Republic of Korea (No. 20134030200350).

\section{REFERENCES}

1. Cady, W. M., Karelitz, M. B., and Turner, L. A., "Radar Scanners and Radomes," $1^{\text {st }}$ Ed., McGraw-Hill, 1948.

2. Moon, S. K., Tan, Y. E., Hwang, J., and Yoon, Y.-J., “Application of 3D Printing Technology for Designing Light-Weight Unmanned Aerial Vehicle Wing Structures,” Int. J. Precis. Eng. Manuf.-Green Tech., Vol. 1, No. 3, pp. 223-228, 2014.
3. Lyu, M.-Y. and Choi, T. G., "Research Trends in Polymer Materials for Use in Lightweight Vehicles," Int. J. Precis. Eng. Manuf., Vol. 16, No. 1, pp. 213-220, 2015.

4. Lee, K.-K., Ro, Y.-C., Kim, Y.-G., Lee, K.-H., and Han, S.-H., "Shape Optimization for Light Weight Design of Direct-Drive Generator in Large-Scale Wind Turbine," Int. J. Precis. Eng. Manuf., Vol. 15, No. 10, pp. 2101-2108, 2014.

5. Bendsoe, M. P. and Sigmund, O., "Topology Optimization: Theory, Methods and Applications," Springer Science \& Business Media, 2003.

6. Choi, H.-J., Mcdowell, D. L., Allen, J. K., and Mistree, F., “An Inductive Design Exploration Method for Hierarchical Systems Design under Uncertainty," Engineering Optimization, Vol. 40, No. 4, pp. 287-307, 2008.

7. Choi, H., McDowell, D. L., Allen, J. K., Rosen, D., and Mistree, F., "An Inductive Design Exploration Method for Robust Multiscale Materials Design," Journal of Mechanical Design, Vol. 130, No. 3, Paper No. 031402, 2008.

8. McDowell, D. L., Panchal, J., Choi, H.-J., Seepersad, C., Allen, J., et al., "Integrated Design of Multiscale, Multifunctional Materials and Products," Butterworth-Heinemann, 2009.

9. Bendsøe, M. P. and Kikuchi, N., "Generating Optimal Topologies in Structural Design Using a Homogenization Method," Computer Methods in Applied Mechanics and Engineering, Vol. 71, No. 2, pp. 197-224, 1988.

10. Saxena, A. and Ananthasuresh, G., "On an Optimal Property of Compliant Topologies," Structural and Multidisciplinary Optimization, Vol. 19, No. 1, pp. 36-49, 2000.

11. Gea, H. C., "Topology Optimization: A New Microstructure-Based Design Domain Method," Computers \& Structures, Vol. 61, No. 5, pp. 781-788, 1996.

12. Wang, M. Y., Wang, X., and Guo, D., "A Level Set Method for Structural Topology Optimization," Computer Methods in Applied Mechanics and Engineering, Vol. 192, No. 1, pp. 227-246, 2003.

13. Jang, S., Park, Y., and Choi, H.-J., "Integrated Design of Aluminum Foam Processing Parameters and Sandwich Panels under Uncertainty," Proc. of the Institution of Mechanical Engineers, Part C: Journal of Mechanical Engineering Science, Paper No. $0954406214558341,2014$.

14. Ashby, M. F. and Cebon, D., "Materials Selection in Mechanical Design,” Le Journal de Physique IV, Vol. 3, No. C7, pp. C7-1-C7-9, 1993.

15. Cressie, N., "Statistics for Spatial Data: Wiley Series in Probability and Statistics," Wiley: New York, 1993.

16. McKay, M. D., Beckman, R. J., and Conover, W. J., "Comparison of Three Methods for Selecting Values of Input Variables in the Analysis of Output from a Computer Code," Technometrics, Vol. 21, No. 2, pp. 239-245, 1979. 